Mario D’Ambrosio, Elisabetta Bigagli*, Lorenzo Cinci, Antonella Gori, Cecilia Brunetti, Francesco Ferrini and Cristina Luceri

\title{
Ethyl acetate extract from Cistus $x$ incanus L. leaves enriched in myricetin and quercetin derivatives, inhibits inflammatory mediators and activates Nrf2/HO-1 pathway in LPS-stimulated RAW 264.7 macrophages
}

https://doi.org/10.1515/znc-2020-0053

Received March 13, 2020; accepted September 8, 2020;

published online October 6, 2020

\begin{abstract}
Cistus $x$ incanus L. is a Mediterranean evergreen shrub used in folk medicine for the treatment of inflammatory disorders but the underlying mechanisms are not fully understood. We therefore investigated the anti-inflammatory effects of an ethyl acetate fraction (EAF) from $C$. $x$ incanus $\mathrm{L}$. leaves on lipopolysaccharide (LPS) activated RAW 264.7 macrophages. HPLC analysis revealed myricetin and quercetin derivatives to be the major compounds in EAF; EAF up to $1 \mu \mathrm{M}$ of total phenolic content, was not cytotoxic and inhibited the mRNA expression of interleukin-6 (IL-6) and
\end{abstract}

Mario D’Ambrosio and Elisabetta Bigagli contributed equally.

*Corresponding author: Elisabetta Bigagli, PhD, Department of NEUROFARBA, University of Florence, Viale Pieraccini 6, 50139 Florence, Italy, E-mail: elisabetta.bigagli@unifi.it. https://orcid.org/ 0000-0003-1892-4343

Mario D'Ambrosio, Lorenzo Cinci and Cristina Luceri, Department of Neuroscience, Psychology, Drug Research and Child Health (NEUROFARBA), Section of Pharmacology and Toxicology, University of Florence, Viale Pieraccini 6, 50139, Florence, Italy. https:// orcid.org/0000-0003-3601-6867 (M. D’Ambrosio). https://orcid.org/ 0000-0002-1799-4828 (L. Cinci). https://orcid.org/0000-0003-12328778 (C. Luceri)

Antonella Gori, Department of Agriculture, Environment, Food and Forestry (DAGRI), University of Florence, Piazzale delle Cascine 18, 50144, Florence, Italy; Institute for Sustainable Plant Protection, National Research Council of Italy (CNR), Via Madonna del Piano 10, Sesto Fiorentino, 50019 Florence, Italy. https://orcid.org/0000-00027304-7526

Cecilia Brunetti, Institute for Sustainable Plant Protection, National Research Council of Italy (CNR), Via Madonna del Piano 10, Sesto Fiorentino, 50019 Florence, Italy. https://orcid.org/0000-0002-85316076

Francesco Ferrini, Department of Agriculture, Environment, Food and Forestry (DAGRI), University of Florence, Piazzale delle Cascine 18, 50144, Florence, Italy. https://orcid.org/0000-0003-2222-0437 cyclooxygenase-2 $(\mathrm{COX}-2)(p<0.05)$ and the production of prostaglandins $\mathrm{E}_{2}\left(\mathrm{PGE}_{2}\right)(p<0.05)$. Meanwhile, EAF triggered the mRNA expression of interleukin-10 (IL-10) and elicited the nuclear translocation of nuclear factor erythroid 2-related factor 2 (Nrf2), as well as the expression of its main target gene, heme oxygenase-1 (HO-1) $(p<0.05)$. These data indicate that EAF attenuates experimental inflammation via the inhibition of proinflammatory mediators and at least in part, by the activation of Nrf2/HO-1 pathway. These effects are likely due to myricetin and quercetin derivatives but the role of other, less abundant components cannot be excluded. Further studies to confirm the relevance of our findings in animal models and to highlight the relative contribution of each component to the anti-inflammatory activity of EAF should be conducted.

Keywords: Cistus x incanus; inflammation; myricitrin; Nrf2; quercetin derivatives; rutin.

\section{Introduction}

Cistus $x$ incanus L. (Pink Rockrose, syn. C. creticus) is a hybrid between $C$. albidus and $C$. crispus, a species of shrubby plant of the Cistaceae family, commonly used in the Mediterranean folk medicine for its anti-inflammatory and skin protective properties [1-4].

Recently, different biological activities have been demonstrated for the leaf extracts of this species and have provided scientific evidence to their traditional utilizations. In particular, antioxidant [5-7], antiviral [8] and antimicrobial properties [9] have been described in experimental models.

Currently, $C$. incanus is considered a medicinal plant and the dried leaves are used as herbal infusions ("Cistus tea”) $[5,10]$ and dietary supplements [11]. In addition, the herbal extract CYSTUS052 ${ }^{\circledR}$ (Dr. Pandalis Urheimische Medizin GmbH und Co. KG, Germany) has given promising 
results as anti-HIV agent [8] and in the treatment of infections of the upper respiratory tract [12]. All these commercial products are especially promoted for their high polyphenolic content [13]. Indeed, leaves of $C$. incanus are rich in proanthocyanidins and flavonols with strong antioxidant activity $[6,14,15]$.

In a cell-free system, we reported that the ethyl acetate fraction (EAF) of $C$. incanus, enriched in flavonols, was the most effective in terms of radical scavenging activity compared to water fractions enriched in proanthocyanidins [6]. However, its potential anti-inflammatory effects have not been yet investigated.

Indeed, besides their antioxidant activity, plantderived phenols may modulate several important components involved in inflammatory pathways and are considered potential complementary sources of bioactive agents for pharmaceutical purposes [16].

Numerous single phenolic compounds such as resveratrol, quercetin and myricetin derivatives have shown anti-inflammatory activities [17-20]. In particular, the ability of polyphenols to reduce inflammation is considered due to their ability to, first, act as antioxidant, second, interfere with oxidative stress signaling and, finally, for their capacity to suppress proinflammatory signaling transductions [21].

However, several evidences also support the hypothesis that the use of phytocomplexes, such as leaf organic extracts has some advantages over the single active ingredients because of the synergism between the different components and the array of biological activities attributable to each different compound [22].

Therefore, the present study was designed to analyze the phytochemical composition of the EAF, to evaluate its anti-inflammatory activity and the underlying molecular mechanisms in lipopolysaccharide (LPS)-activated RAW 264.7 macrophages, an experimental model of inflammation.

\section{Material and methods}

\subsection{Preparation of EAF and quantification of polyphenols by HPLC-DAD}

C. incanus leaves were collected from plants growing on seashore dunes in Southern Tuscany $\left(42^{\circ} 46^{\circ} \mathrm{N}, 10^{\circ} 53^{\prime} \mathrm{E}\right)$ in July 2017 by CB and AG and identified by FF and Prof. Bussotti (Department of Agriculture, Environment, Food and Forestry [DAGRI]), University of Florence, Italy.

Harvested plant material was frozen in liquid nitrogen and stored at $-80^{\circ} \mathrm{C}$. EAF was obtained following the same extraction procedure reported by Gori et al. (2016) [6]. In this protocol, EAF was obtained from the crude ethanolic extract of $C$. incanus leaves through a liquidliquid partitioning with ethyl acetate and water. EAF was then analyzed with a Perkin Elmer Flexar liquid chromatograph equipped with a quaternary 200Q/410 pump and a LC 200 diode array detector (Perkin Elmer Bradford, CT, USA). Quantification of the single phenolic compounds was directly performed by HPLC-DAD. In particular, six individual compounds, i.e. gallic acid, epicatechin, myricetin 3-O-rhamnoside, quercetin 3-O-rhamnoside and rutin, were quantified with their own four-point regression standard curves. Calibration of epicatechin was performed at $280 \mathrm{~nm}$ using epicatechin as reference compound. Calibration of myricetin and kaempferol derivatives was performed at $350 \mathrm{~nm}$ using myricetin 3-O-rhamnoside and kaempferol 7-O-glucoside as reference compounds, respectively. Analytical separation was performed on a reversed-phase Waters Nova-Pak C18 column $(4.9 \times 250 \mathrm{~mm}, 4 \mu \mathrm{m})$ (Water Milford, MA, USA), operating at $30^{\circ} \mathrm{C}$. The mobile phase consisted of $1 \%$ aqueous formic acid (solvent A) and $1 \%$ formic acid in acetonitrile (solvent $\mathrm{B}$ ). The elution gradient consisted of $2 \% \mathrm{~B}$ isocratic for $10 \mathrm{~min}$, from 2 to 98\% B linear for $30 \mathrm{~min}, 98 \% \mathrm{~B}$ isocratic for $7 \mathrm{~min}$, then starting condition during $5 \mathrm{~min}$ to re-equilibrate the column. The flow rate was $0.6 \mathrm{~mL} / \mathrm{min}$ and the injection volume was $10 \mu \mathrm{L}$.

\subsection{Cell cultures}

RAW 264.7, a murine macrophage cell line obtained from American Type Culture Collection (Rockville, USA), was cultured in Dulbecco's modified eagle medium (DMEM) (Euroclone, Milan, Italy) supplemented with 10\% fetal bovine serum (FBS) (Carlo Erba reagents, Milan, Italy), $1 \%$ L-glutamine (Carlo Erba reagents, Milan, Italy) and $1 \%$ Penicillin/Streptomycin (Carlo Erba reagents, Milan, Italy) at $37^{\circ} \mathrm{C}$ in an atmosphere containing $5 \% \mathrm{CO}_{2}$.

\subsection{Cell viability}

RAW 264.7 cells were seeded in 96-well plates at a density of $5 \times 10^{3}$ cells/well in $100 \mu \mathrm{L}$ of medium added of $10 \%$ FBS. After $24 \mathrm{~h}$ incubation at $37^{\circ} \mathrm{C}$ in $5 \% \mathrm{CO}_{2}, \mathrm{EAF}(0.125-25 \mu \mathrm{g} / \mathrm{mL})$ was added to the wells and incubated for $24 \mathrm{~h}$ at $37^{\circ} \mathrm{C}$ in $5 \% \mathrm{CO}_{2}$. Cell viability was assessed by the colorimetric method based on [3-(4,5-dimethylthiazol2-yl)-5-(3-carboxymethoxyphenyl)-2-(4-sulfophenyl)-2H-tetrazolium, inner salt; MTS] (Promega Corporation, Madison, USA). The optical density of the chromogenic product was measured at $490 \mathrm{~nm}$. Data were expressed as percentage of viable cells compared to untreated cells.

\subsection{LPS-induced inflammation in RAW 264.7}

RAW 264.7 cells were seeded in a 24-well microplate at a density of $1 \times 10^{5}$ cells/well for $24 \mathrm{~h}$. The cells were then exposed to LPS $(1 \mu \mathrm{g} / \mathrm{mL})$ (Sigma Aldrich, Milan, Italy) alone or in the presence of EAF at concentration ranging from 0.25 to $1 \mu \mathrm{M}$ of total phenolic content. Indomethacin $10 \mu \mathrm{M}$ and celecoxib $3 \mu \mathrm{M}$ (Sigma Aldrich, Milan, Italy), were used as reference anti-inflammatory drugs (positive controls). After $18 \mathrm{~h}$ at $37^{\circ} \mathrm{C}$, cell lysates were collected for gene expression analyses and supernatants for nitric oxide (NO) and prostaglandins $\mathrm{E}_{2}\left(\mathrm{PGE}_{2}\right)$ determination. 


\subsection{Determination of NO production}

NO release was measured with Griess reagent $(1 \%[\mathrm{w} / \mathrm{v}]$ sulfanilamide and $0.1 \%[\mathrm{w} / \mathrm{v}] \mathrm{N}-[1$-naphthyl] ethylenediamine hydrochloride in $2.5 \%$ [v/v] phosphoric acid) [18]. $100 \mu \mathrm{L}$ of the cell supernatant was mixed with an equal volume of Griess reagent and incubated at room temperature (RT) for $30 \mathrm{~min}$. The absorbance was measured at $540 \mathrm{~nm}$ using a VICTOR 3 Wallac 1421 (Perkin Elmer, Ramsey, USA) and NO production calculated with reference to a standard curve obtained with $\mathrm{NaNO}_{2}$.

\section{6 $\mathrm{PGE}_{2}$ determination}

$\mathrm{PGE}_{2}$ levels were measured in the supernatants, using an ELISA kit (Cayman, Ann Arbor, USA) according to the manufacturer's specifications.

\subsection{Reverse transcriptase-polymerase chain reaction (RT-PCR)}

Total RNA was extracted from RAW 264.7 using the NucleoSpin ${ }^{\circledR}$ RNA (Macherey-Nagel, Bethlehem, USA). For first-strand cDNA synthesis, $100 \mathrm{ng}$ of total RNA from each sample was reverse-transcribed using the Revert Aid RT Kit (Thermo Scientific, Waltham, USA). Primers were designed on the basis of the mouse GenBank sequences (Supplementary Table 1). For each target gene, the relative amount of mRNA in the samples was calculated as the ratio of each gene to ribosomal protein large P1 (RPLP-1) mRNA [18].

\subsection{Immunocytofluorescence for nuclear factor erythroid 2-related factor 2 (Nrf2) localization}

RAW 264.7 cells were grown in poly-D-lysine-coated glass dishes for $24 \mathrm{~h}$, then treated with LPS $(1 \mu \mathrm{g} / \mathrm{mL})$, and EAF $0.5 \mu \mathrm{g} / \mathrm{mL}$ for $18 \mathrm{~h}$. Cells were then fixed with cold $4 \%(\mathrm{w} / \mathrm{v})$ paraformaldehyde for $20 \mathrm{~min}$, rehydrated in PBS for $15 \mathrm{~min}$ and permeabilized in $0.1 \%(\mathrm{w} / \mathrm{v})$ TritonX100 at RT for $10 \mathrm{~min}$. After being washed with PBS, the cells were blocked for unspecific fluorescence with 3\% bovine serum albumin (BSA) for $1 \mathrm{~h}$ and then incubated with Rabbit anti-nuclear factor erythroid 2-related factor 2 (Nrf2) polyclonal antibody C-20 (1:300) (Santa Cruz Biotechnology, Dallas, USA) at $4{ }^{\circ} \mathrm{C}$ overnight followed by the fluorescent secondary antibodies AlexaFluor 594 goat anti-rabbit (1:333) (Invitrogen, San Giuliano Milanese, Italy). Cells were also counterstained with 4',6-diamidin-2-phenylindole (DAPI) to show the nuclear translocation [18].

\subsection{Image acquisition and analysis}

Microscopic analysis was performed with an Olympus BX63 microscope equipped with a Metal Halide Lamp (Prior Scientific Instruments Ltd., Cambridge, United Kingdom) and a digital camera Olympus XM 10 (Olympus, Milan, Italy). A total of 10 photomicrographs were randomly taken for each sample (average number of cells/field: 100; average number of analyzed cells in total: 1000) and the number of cells with Nrf2 nuclear translocation was counted using ImageJ 1.33 image analysis software (http://rsb.info.nih.gov/ij).

\subsection{Statistical analysis}

Data were analyzed by one-way analysis of variance (ANOVA) with Dunnett's multiple comparisons and expressed as means \pm standard error (SEM) of four independent experiments. All analyses were carried out using GraphPad Prism 7.0 (GraphPad Software, San Diego, USA). A $p$-value $>0.05$ was considered significant.

\section{Results}

\subsection{Qualitative and quantitative characterization of polyphenolic compounds in EAF}

The HPLC characterization of EAF, according to our previous investigation [6], revealed the presence of four classes of polyphenols: gallic acid, catechin derivatives, proanthocianidins and flavonols (myricetin derivatives, quercetin derivatives and kaempferol derivatives) (Table 1). The identification of single compounds was performed comparing the UV-VIS spectra and the retention time with those of authentic standards. Overall, our results showed that EAF was rich in total phenolic content (about $83 \mathrm{mM}$ ) and that myricitrin and rutin were the most abundant compounds (Table 1).

\subsection{Effect of EAF on RAW 264.7 cell viability}

Exposure of RAW 264.7 to different EAF concentrations ranging from 10 to $50 \mu \mathrm{M}$ of total phenols for $24 \mathrm{~h}$ resulted in a concentration-dependent reduction of cell viability,

Table 1: HPLC-DAD quantification of main flavonols in EAF.

\begin{tabular}{lr}
\hline Compounds & $\mathrm{mM}$ \\
\hline Gallic acid & $1.10 \pm 0.09$ \\
(-)-Gallocatechin and (-)-epigallocatechin & $2.19 \pm 0.26$ \\
Proanthocyanidin polymers & $6.34 \pm 0.81$ \\
Myricetin-3-O-hexoside & $0.10 \pm 0.01$ \\
Myricetin 3-O-rhamnoside (Myricitrin) & $27.0 \pm 1.94$ \\
Other Myricetin derivatives & $1.86 \pm 0.19$ \\
Total myricetin derivates & $\mathbf{2 8 . 9 6} \pm \mathbf{0 . 2 1}$ \\
Quercetin-3-O-pentoside & $3.80 \pm 0.23$ \\
Quercetin 3-O-rhamnoside (Quercitrin) & $6.70 \pm 0.56$ \\
Other quercetin derivatives & $14.6 \pm 1.23$ \\
Quercetin 3-O-rutinoside (Rutin) & $19.0 \pm 1.45$ \\
Total quercetin derivates & $\mathbf{4 4 . 1 0 \pm 0 . 3 0}$ \\
Kaempferol 3-O-rutinoside & $0.08 \pm 0.01$ \\
Kaempferol-3-(3",6"-dicoumaroyl)-glucose & $0.11 \pm 0.01$ \\
\hline
\end{tabular}

Data are mean \pm SD of three replicates. 
whereas concentrations from 0.25 to $1 \mu \mathrm{M}$ were not toxic (data not shown). Based on these results, concentrations of $0.25,0.5$ and $1 \mu \mathrm{M}$ were chosen as the nontoxic range for further anti-inflammatory analysis.

\subsection{Effects of EAF on LPS-induced NO production}

LPS treatment resulted in a significant increase in NO production that was concentration dependently reduced by EAF. In particular, EAF at $1 \mu \mathrm{M}$ of total phenolic content, reduced NO production by about $50 \%$ compared with LPS $(p<0.001)$ and it exerted slight, but significant effects also at 0.5 and $0.25 \mu \mathrm{M}(p<0.05$ and $p<0.01$ respectively). Celecoxib, a selective inhibitor of COX-2, markedly reduced NO production by more than $70 \%$ $(p<0.001)$. On the contrary, the effect of indomethacin was lower than that of celecoxib and comparable to that of EAF $0.25 \mu \mathrm{M}$ (Figure 1).

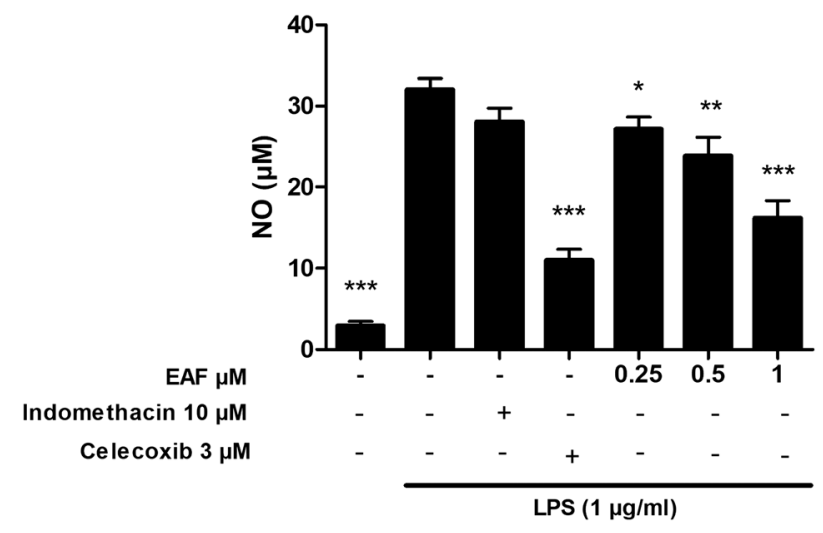

Figure 1: Effect of ethyl acetate fraction (EAF) on NO production in RAW 264.7 stimulated with LPS for 18 h. ${ }^{*} p<0.05$; ${ }^{\star \star} p<0.01$; $\star \star \star p<0.001$ vs LPS by one-way ANOVA and Dunnett's multiple comparisons test. Data are expressed as mean \pm SEM of four replicates.

\subsection{Effect of EAF on LPS-induced expression of proinflammatory cytokines and enzymes}

As shown in Table 2, LPS, after $18 \mathrm{~h}$ of treatment markedly induced the mRNA expression of IL- 6 and reduced that of interleukin-10 (IL-10) compared to control cells. These effects were significantly counteracted by $\operatorname{EAF} 1 \mu \mathrm{M}(p<0.001)$ but to a lower extent compared to indomethacin $10 \mu \mathrm{M}$ and celecoxib $3 \mu \mathrm{M}$. Moreover, compared to LPS alone, both the anti-inflammatory drugs, indomethacin and celecoxib, used as positive controls, significantly reduced the expression of inducible nitric oxide synthase (iNOS), tumor necrosis factor alpha (TNF- $\alpha$ ) and interleukin 1 beta (IL-1 $\beta$ ) whereas EAF $1 \mu \mathrm{M}$ had no effect. EAF at lower concentrations did not exert any significant effect (data not shown).

\subsection{Effect of EAF on LPS-induced COX-2 expression and $\mathrm{PGE}_{2}$ production}

LPS treatment significantly increased the mRNA expression of COX-2 and $\mathrm{PGE}_{2}$ production compared to control cells $(p<0.001)$; upon treatment with EAF at $1 \mu \mathrm{M}, \mathrm{COX}-2$ expression and $\mathrm{PGE}_{2}$ production were significantly attenuated $(p<0.001)$ even if to a lower extent compared to indomethacin and celecoxib (Figure 2, Panel A and B). EAF at lower concentrations $(0.5$ and $0.25 \mu \mathrm{M})$, did not exert any significant effect either on COX-2 expression and $\mathrm{PGE}_{2}$ production.

\subsection{Effects of EAF on nuclear translocation of Nrf2 and HO-1 mRNA expression}

In cells exposed to LPS, the percentage of cells positive for nuclear Nrf2 staining was significantly reduced compared to control cells $(p<0.01)$ (Figure 3, panels A, B and D). On the contrary, the treatment with EAF $1 \mu \mathrm{M}$ restored nuclear Nrf2 expression to levels similar of those of the control cells $(p<0.01)$ (Figure 3, panel C, and D).

Table 2: Effect of EAF on iNOS, IL-6, IL-10, TNF $\alpha$ and IL-1 $\beta$ mRNA expression in LPS-stimulated RAW 264.7 cells.

\begin{tabular}{lccccc}
\hline & Control & LPS $1 \mu \mathrm{g} / \mathrm{mL}$ & Indomethacin 10 $\mu$ M & Celecoxib 3 $\mu$ M & EAF 1 $\mu$ M \\
\hline iNOS & $0.16 \pm 0.16^{\star \star}$ & $0.85 \pm 0.25$ & $0.54 \pm 0.01^{\star}$ & $0.37 \pm 0.04^{\star \star}$ & $0.68 \pm 0.22$ \\
IL-6 & $0.23 \pm 0.02^{\star \star \star}$ & $0.74 \pm 0.03$ & $0.07 \pm 0.01^{\star \star \star}$ & $0.06 \pm 0.01^{\star \star \star}$ & $0.42 \pm 0.02^{\star \star \star}$ \\
IL-10 & $0.42 \pm 0.03^{\star \star}$ & $0.26 \pm 0.01$ & $0.66 \pm 0.02^{\star \star \star}$ & $0.71 \pm 0.03^{\star \star \star}$ & $0.50 \pm 0.01^{\star \star \star}$ \\
TNF $\alpha$ & $0.23 \pm 0.04^{\star \star}$ & $0.74 \pm 0.05$ & $0.21 \pm 0.01^{\star \star \star}$ & $0.20 \pm 0.03^{\star \star \star}$ & $0.71 \pm 0.06$ \\
IL-1 $\beta$ & $0.36 \pm 0.01^{\star \star \star}$ & $0.99 \pm 0.05$ & $0.29 \pm 0.01^{\star \star \star}$ & $0.31 \pm 0.02^{\star \star \star}$ & $0.96 \pm 0.01$ \\
\hline
\end{tabular}

Data are expressed as mean \pm SEM of four replicates; ${ }^{* \star *} \mathrm{p}<0.001$; ${ }^{* \star} \mathrm{p}<0.01$; ${ }^{*} \mathrm{p}<0.05$ vs LPS by one-way ANOVA and Dunnett's multiple comparisons test. 
A

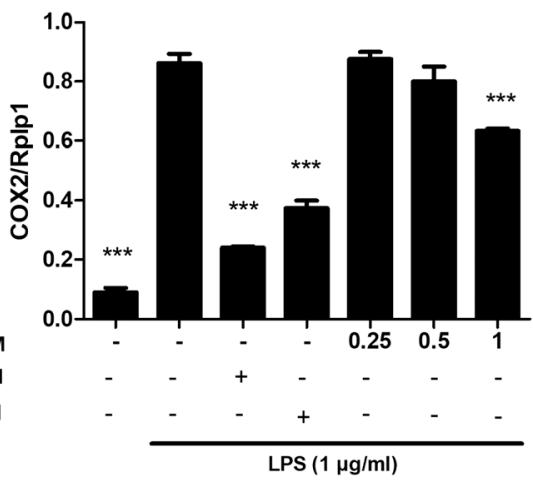

B

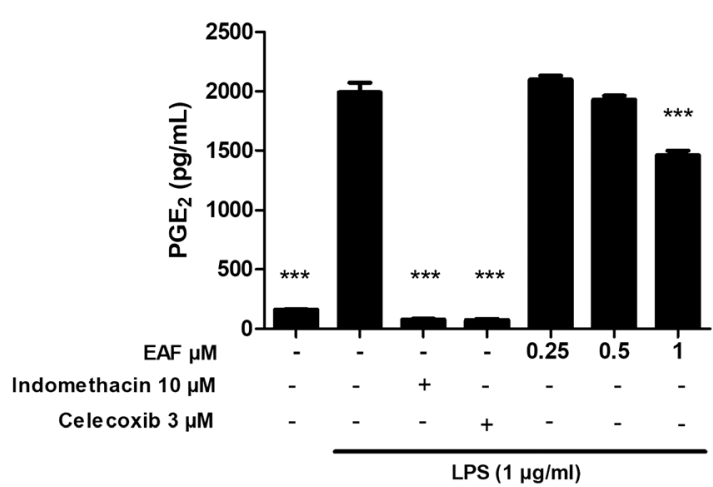

Figure 2: Effect of Ethyl acetate fraction (EAF) on COX-2 mRNA expression (A) and PGE 2 production (B) in RAW 264.7 stimulated with LPS for $18 \mathrm{~h}$. ${ }^{\star \star} p<0.001 \mathrm{vs}$ LPS, by one-way ANOVA and Dunnett's multiple comparisons test. Data are expressed as mean \pm SEM of four replicates.
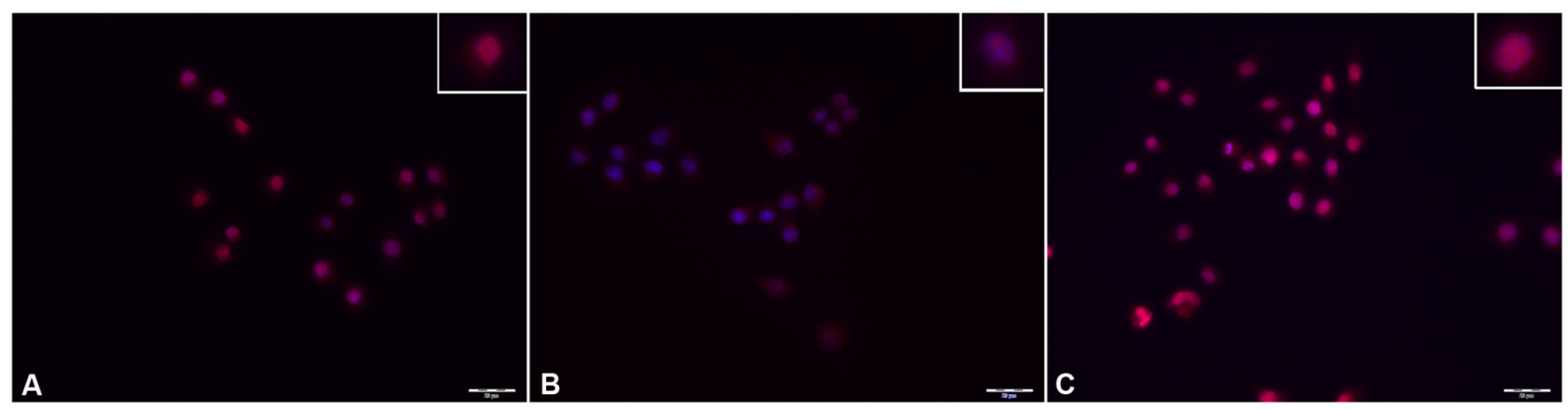

D

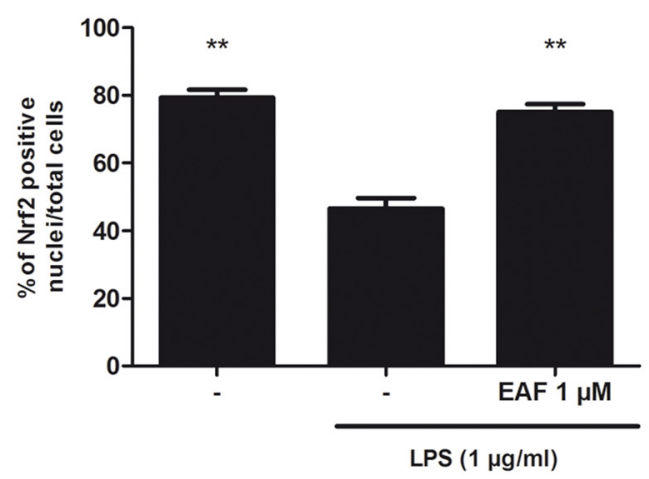

E

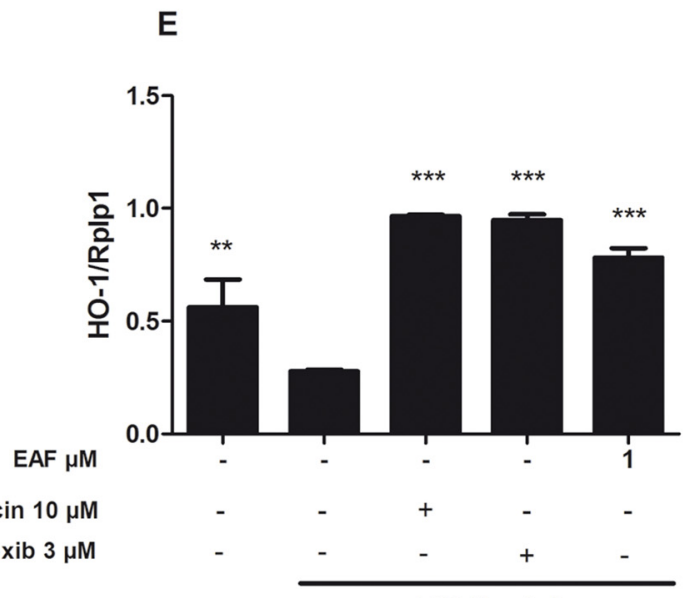

Indome thacin $10 \mu \mathrm{M}$

Celecoxib $3 \mu \mathrm{M}$

LPS $(1 \mu \mathrm{g} / \mathrm{ml})$

Figure 3: Upper panel: Nrf2 localization in unstimulated RAW 264.7 (CTRL) (Panel A), RAW 264.7 stimulated with LPS for $18 \mathrm{~h}$ (LPS) (Panel B) and in RAW 264.7 stimulated with LPS and treated with EAF, $1 \mu \mathrm{M}$ of total phenolic content (Panel C). The nuclear translocation of Nrf2 was immunostained with Nrf2 antibody (red). DAPI was used to visualize the nuclei (blue) and the merged Nrf2 (red)/DAPI (blue) image is shown in dark pink. Top right insets show higher magnification of illustrative cells. Scale bar $20 \mu \mathrm{m}$. Lower panel: percentage of Nrf2 positive nuclei (Panel D) HO-1 mRNA expression in RAW 264.7 stimulated with LPS for $18 \mathrm{~h}$ (Panel E). ${ }^{\star \star \star} p<0.001$; ${ }^{\star \star} p<0.01$ vs LPS, by one-way ANOVA and Dunnett's multiple comparisons test. Data are expressed as mean \pm SEM of four replicates. 
To further explore the effects of Nrf2 nuclear translocation, changes in the expression of its main downstream target gene heme oxygenase-1 (HO-1) were investigated. As shown in Figure 3 panel E, the mRNA expression of HO-1 was significantly reduced in LPS treated cells compared to control cells $(p<0.01)$; EAF $1 \mu \mathrm{M}$ restored the mRNA expression of HO-1 at levels even above those of the control cells, and similar to indomethacin and celecoxib.

\section{Discussion}

Recent evidence suggests that the biological activities of flavonoids go beyond their ability to counteract oxidative stress, being able to regulate signal transduction pathways, transcription factors, gene expression and microRNA $[18,23]$.

Our results demonstrate that EAF inhibited the LPS-induced production of $\mathrm{PGE}_{2}$ by suppressing the transcription of COX-2. EAF also reduced the mRNA expression of the proinflammatory cytokine IL- 6 and elicited that of the anti-inflammatory cytokine IL-10.

These beneficial effects were accompanied by the activation of the transcription factor Nrf2 that plays an important role in cellular defense against oxidative stress [24] and inflammation [18]. The activation of Nrf2 by EAF, resulted in the transcriptional upregulation of HO-1, one of its main target genes, also involved in the inhibition of LPS-induced NO production [25]. This is consistent with the positive effect of EAF against LPS-induced production of NO and may explain that, although we did not observe significant effects of EAF on iNOS expression, the transcriptional control of HO-1 may have played a role. EAF also reduced the expression of IL- 6 and this effect may be mediated by Nrf2 activation since in in vitro and in vivo models of inflammation, Kobayashi et al. [26] reported that the transcriptional control of IL-6 is regulated by Nrf2.

It is also important to note that although EAF was less effective in reducing proinflammatory mediators compared to celecoxib and indomethacin, its effects were detected at a lower concentration compared to the reference anti-inflammatory drugs and involved similar pathways beyond the inhibition of $\mathrm{COX}-2$ and $\mathrm{PGE}_{2}$ : both celecoxib and indomethacin in fact, were reported to activate NRF2/HO-1 pathway [27-29].

Plants from Mediterranean-type ecosystems are challenged by environmental stressors such as drought, high solar irradiance and high temperature. These conditions induce the activation of antioxidant defense systems including the biosynthesis of antioxidant phenols. As other plants from Mediterranean area [30], C. $x$ incanus L leaves contain high concentrations of polyphenols and the EAF, in particular, results enriched in flavonols and exhibit a high antiradical activity [6].

Our phytochemical characterization demonstrated that myricetin and quercetin derivatives were the main components of EAF and several data support their antiinflammatory activity; myricitrin, a myricetin glycoside (myricetin-3-O-rhamnoside), $(25 \mu \mathrm{g} / \mathrm{mL})$ inhibited advanced glycation end products (AGE)-induced inflammation and oxidative stress in cardiomyocytes via the regulation of nuclear factor-kB (NF-kB) and Nrf2/HO-1 signaling [31]. Myricitrin (100-400 $\mu \mathrm{g} / \mathrm{mL})$ also inhibited NO production, IL- 6 and TNF $\alpha$ but failed to reduce $\mathrm{PGE}_{2}$ in RAW 264.7 [32]. In an in vivo model of cisplatin-induced inflammatory response, myricetin, dose-dependently, attenuated the production of markers of inflammation such as NF-kB, IL-6 and TNF-a, and restored Nrf-2 levels [33].

The other main component of EAF is rutin, the glycosidic form of quercetin. Rutin $200 \mu \mathrm{M}$ suppressed palmitic acid-triggered production of ROS and TNFa in RAW 264.7 [34] and at concentrations of $2.5-10 \mu \mathrm{g} / \mathrm{mL}$, suppressed IL-6 production via down regulation of NF- $\mathrm{kB}, \mathrm{COX}-2$ and iNOS in LPS and IFN- $\gamma$-stimulated RAW 264.7 [35]. The effects of quercetin and its derivatives on inflammation have been also largely investigated in preclinical models as reviewed in [36, 37]. For instance, in LPS-stimulated RAW 264.7, quercetin $12.5 \mu \mathrm{M}$ reduced NO and IL-6 production and inhibited iNOS expression and NF-kB activation [38]; similarly, quercetin-3-O-(200-gallate)-a-L-rhamnopyranoside $(0.2-2 \mu \mathrm{g} / \mathrm{mL})$, a quercetin derivative, in RAW 264.7 stimulated with peptidoglycan, reduced NO production and COX-2 and iNOS expression [39].

Since myricetin and quercetin derivatives are the most abundant compounds found in EAF extract, we hypothesized that they are likely responsible for the effects observed in our study; however, it is important to note that although literature data support their anti-inflammatory activity, when myricitrin and rutin were tested as single compounds, the anti-inflammatory effects were observed at much higher concentrations (in the range of $50-400 \mu \mathrm{M}$ for myricitrin and in the range of $3-16 \mu \mathrm{M}$ for rutin) compared to those employed in the present study.

We in fact, selected concentrations that did not affect cell viability and that are likely reachable in vivo, due to the low bioavailability of these compounds. In our study, myricetrin and rutin, representing 34.6 and $23.5 \%$ of the EAF phenolic components, had in fact a final concentrations of $156 \mathrm{ng} / \mathrm{mL}(0.336 \mu \mathrm{M})$ and $106 \mathrm{ng} / \mathrm{mL}(0.174 \mu \mathrm{M})$, respectively. It is possible that the anti-inflammatory effects of EAF depend on additive effects or synergism between these two main compounds but the contribution of 
other components of the EAF phytocomplex, cannot be excluded.

Interestingly, the beneficial health effects of $C$. incanus extracts have been demonstrated in humans in two clinical studies: 7 days treatment with tablets containing $C$. incanus $\left(\right.$ CYSTUS052 ${ }^{\circledR}$ ) extract (approximately $220 \mathrm{mg}$ of polyphenols per day) decreased the symptoms of influenza and the levels of the inflammatory marker C-reactive protein in patients with upper respiratory tract infections [12].

The supplementation with $C$. incanus herbal tea enriched in myricetin, quercetin and isoquercetin exceeding (exceeding $800 \mu \mathrm{g} / \mathrm{g} \mathrm{dw}$ ) for 12 weeks, reduced oxidative stress markers and improved the lipid profile in healthy adults [10].

In conclusion, we demonstrated that nontoxic concentrations of EAF total phenolic content, reduces experimental inflammation via the inhibition of proinflammatory mediators and, at least in part, by the activation of Nrf2/ HO-1 pathway. Although the in vivo bioactivity of EAF and the relative contribution of each component should be evaluated, these results provide the scientific support of its potential anti-inflammatory effects.

Acknowledgment: This research was supported by the University of Florence (Fondi di Ateneo) and by funds from the Italian MIUR to CNR, project NUTR-AGE (FOE-2019, DSB.AD004.271).

Author contributions: CL designed the experiments; CL and $\mathrm{EB}$ analyzed and interpreted the data. MD and $\mathrm{LC}$ performed the experiments. AG, CB and FF prepared and characterized the extract. All authors read and approved the final manuscript.

Research funding: This study was supported by the University of Florence and funds from the Italian MIUR to CNR project NUTR-AGE (FOE-2019, DSB.AD004.271)

Conflict of interest: The authors show no conflict of interests.

\section{Supplementary Table 1.}

Primer sequences

\section{References}

1. Petereit F, Kolodziej H, Nahrstedt A. Flavan-3-ols and proanthocyanidins from Cistus incanus. Phytochemistry 1991;30: 981-5.

2. Danne A, Petereit F, Nahrstedt A. Proanthocyanidins from Cistus incanus. Phytochemistry 1993;34:1129-33.

3. Barrajón-Catalán E, Fernández-Arroyo S, Roldán C, Guillén E, Saura D, Segura-Carretero A, et al. A systematic study of the polyphenolic composition of aqueous extracts deriving from several Cistus genus species: evolutionary relationship. Phytochem Anal 2011;22:303-12.

4. Guzmán B, Vargas P. Systematics, character evolution, and biogeography of Cistus L. (Cistaceae) based on ITS, trnL-trnF, and matK sequences. Mol Phylogenet Evol 2005;37:644-60.

5. Riehle P, Vollmer M, Rohn S. Phenolic compounds in Cistus incanus herbal infusions - antioxidant capacity and thermal stability during the brewing process. Food Res Int 2013;53:891-9.

6. Gori A, Ferrini F, Marzano MC, Tattini M, Centritto M, Baratto MC, et al. Characterization and antioxidant activity of crude extract and polyphenolic rich fractions from $C$. incanus leaves. Int J Mol Sci 2016;17:e1344.

7. Dimcheva V, Karsheva V. Cistus incanus from Strandja mountain as a source of bioactive antioxidants. Plants 2018;26:e8.

8. Rebensburg A, Helfer M, Schneider M, Koppensteiner H, Eberle J, Schindler M, et al. Potent in vitro antiviral activity of Cistus incanus extract against HIV and Filoviruses targets viral envelope proteins. Sci Rep 2016;6:20394.

9. Tomás-Menor L, Morales-Soto A, Barrajón-Catalán E, RoldánSegura C, Segura-Carretero A, Micol V. Correlation between the antibacterial activity and the composition of extracts derived from various Spanish Cistus species. Food Chem Toxicol 2013;55: 313-22.

10. Kuchta A, Konopacka A, Waleron K, Viapiana A, Wesołowski M, Dąbkowski K, et al. The effect of Cistus incanus herbal tea supplementation on oxidative stress markers and lipid profile in healthy adults. Cardiol J 2019. https://doi.org/10.5603/C). a2019.0028 [Epub ahead of print].

11. Cacak-Pietrzak G, Różyło R, Dziki D, Gawlik-Dziki U, Sułek A, Biernacka B. Cistus incanus L. as an innovative functional additive to wheat bread. Foods 2019;8:e349.

12. Kalus U, Grigorov A, Kadecki O, Jansen JP, Kiesewetter H, Radtke H. Cistus incanus (CYSTUS052) for treating patients with infection of the upper respiratory tract: a prospective, randomised, placebo-controlled clinical study. Antivir Res 2009;84:267-71.

13. Jeszka-Skowron M, Zgoła-Grześkowiak A, Frankowski R. Cistus incanus a promising herbal tea rich in bioactive compounds: LC-MS/MS Determination of catechins,

\begin{tabular}{llll}
\hline Gene & Primer forward & Primer reverse & Base pair \\
\hline RPLP-1 & ATCTACTCCGCCCTCATCCT & CAGATGAGGCTCCCAATGTT & 155 \\
COX-2 & TCCTCCTGGAACATGGACTC & CCCCAAAGATAGCATCTGGA & 321 \\
iNOS & AGACCTCAACAGAGCCCTCA & GCAGCCTCTTGTCTTGACC & 305 \\
HO-1 & GGCTGCCCTGGAGCAGGAC & AGGTCACCCAGGTAGCGGG & 165 \\
IL-10 & AGGCGCTGTCATCGATTCTC & AGGAAGAACCCCTCCCATCA & 489 \\
IL-6 & TCCTCTCTGCAAGAGACTTCC & TCCTCTCTGCAAGAGACTTCC & 513 \\
TNF $\alpha$ & TAGCCCACGTCGTAGCAAC & ACCCTGAGCCATAATCCCCT & 566 \\
IL-1 $\beta$ & CAGGCAGGCAGTATCACTCA & AGGCCACAGGTATTTTTCG & 350 \\
\hline
\end{tabular}


flavonols, phenolic acids and alkaloids-a comparison with Camellia sinensis, Rooibos and Hoan ngoc herbal tea. J Food Compos Anal 2018;74:71-81.

14. Santagati NA, Salerno L, Attaguile G, Savoca F, Ronsisvalle G. Simultaneous determination of catechins, rutin, and gallic acid in cistus species extracts by HPLC with diode array detection. J Chromatogr Sci 2008;46:150-6.

15. Wittpahl G, Kölling-Speer I, Basche S, Herrmann E, Hannig M, Speer K, et al. The polyphenolic Composition of Cistus incanus herbal tea and its antibacterial and anti-adherent activity against Streptococcus mutans. Planta Med 2015;81:1727-35.

16. Hussain T, Tan B, Yin Y, Blachier F, Tossou MCB, Rahu N. Oxidative stress and inflammation: what polyphenols can do for us? Oxid Med Cell Longev 2016;2016:1-9. e7432797.

17. Cho BO, Yin HH, Park SH, Byun EB, Ha HY, Jang SI. Antiinflammatory activity of myricetin from Diospyros lotus through suppression of NF-KB and STAT1 activation and Nrf2-mediated HO-1 induction in lipopolysaccharide-stimulated RAW264.7 macrophages. Biosci Biotechnol Biochem 2016;80: 1520-30.

18. Bigagli E, Cinci L, Paccosi S, Parenti A, D’Ambrosio M, Luceri C. Nutritionally relevant concentrations of resveratrol and hydroxytyrosol mitigate oxidative burst of human granulocytes and monocytes and the production of pro-inflammatory mediators in LPS-stimulated RAW 264.7 macrophages. Int Immunopharm 2017;43:147-55.

19. Hou W, Hu S, Su Z, Wang Q, Meng G, Guo T, et al. Myricetin attenuates LPS-induced inflammation in RAW 264.7 macrophages and mouse models. Future Med Chem 2018;10: 2253-64.

20. Lee HN, Shin SA, Choo GS, Kim HJ, Park YS, Kim BS, et al. Antiinflammatory effect of quercetin and galangin in LPS-stimulated RAW264.7 macrophages and DNCB-induced atopic dermatitis animal models. Int J Mol Med 2018;41:888-98.

21. Zhang H, Tsao R. Dietary polyphenols, oxidative stress and antioxidant and anti-inflammatory effects. Curr Opin Food Sci 2016;8:33-42.

22. Ferlazzo N, Cirmi S, Calapai G, Ventura-Spagnolo E, Gangemi S, Navarra M. Anti-inflammatory activity of citrus bergamia derivatives: where do we stand? Molecules 2016;21:e1273.

23. Vezza T, Rodríguez-Nogales A, Algieri F, Utrilla MP, RodriguezCabezas ME, Galvez J. Flavonoids in inflammatory bowel disease: a review. Nutrients 2016;8:211-33.

24. Silva-Islas CA, Maldonado PD. Canonical and non-canonical mechanisms of Nrf2 activation. Pharmacol Res 2018;134:92-9.

25. Lin HY, Juan SH, Shen SC, Hsu FL, Chen YC. Inhibition of lipopolysaccharide-induced nitric oxide production by flavonoids in RAW264. 7 macrophages involves heme oxygenase-1. Biochem Pharmacol 2003;66:1821-32.
26. Kobayashi EH, Suzuki T, Funayama R, Nagashima T, Hayashi M, Sekine $\mathrm{H}$, et al. $\mathrm{Nrf} 2$ suppresses macrophage inflammatory response by blocking proinflammatory cytokine transcription. Nat Commun 2016;7:e11624.

27. Wang JS, Ho FM, Kang HC, Lin WW, Huang KC. Celecoxib induces heme oxygenase- 1 expression in macrophages and vascular smooth muscle cells via ROS-dependent signaling pathway. Naunyn-Schmiedeberg's Arch Pharmacol 2011;383:159-68.

28. Yoshinaga N, Arimura N, Otsuka H, Kawahara KI, Hashiguchi T, Maruyama I, et al. NSAIDs inhibit neovascularization of choroid through HO-1-dependent pathway. Lab Invest 2011;91:1277-90.

29. Al-Rashed F, Calay D, Lang M, Thornton CC, Bauer A, Kiprianos A, et al. Celecoxib exerts protective effects in the vascular endothelium via COX-2-independent activation of AMPK-CREBNrf2 signaling. Sci Rep 2018;8:e6271.

30. Santagostini L, Caporali E, Giuliani C, Bottoni M, Ascrizzi R, Araneo SR, et al. Humulus lupulus L. cv. Cascade grown in Northern Italy: morphological and phytochemical characterization. Plant Biosys 2020;154:316-25.

31. Zhang B, Shen Q, Chen Y, Pan R, Kuang S, Liu G, et al. Myricitrin alleviates oxidative stress-induced inflammation and apoptosis and protects mice against diabetic cardiomyopathy. Sci Rep 2017;7:e44239.

32. Qi S, Feng Z, Li Q, Qi Z, Zhang Y. Myricitrin modulates NADPH oxidase-dependent ROS production to inhibit endotoxinmediated inflammation by blocking the JAK/STAT1 and NOX2/p47 phox pathways. Oxid Med Cell Longev 2017;2017:e9738745.

33. Rehman MU, Rather IA. Myricetin abrogates Cisplatin-induced oxidative stress, inflammatory response, and goblet cell disintegration in colon of wistar rats. Plants 2019;9:e28.

34. Gao M, Ma Y, Liu D. Rutin suppresses palmitic acids-triggered inflammation in macrophages and blocks high fat diet-induced obesity and fatty liver in mice. Pharm Res 2013;30:2940-50.

35. Choi SY, Choi JY, Lee JM, Lee S, Cho EJ. Tartary buckwheat on nitric oxide-induced inflammation in RAW264.7 macrophage cells. Food and Funct 2015;6:2664-70.

36. Li Y, Yao J, Han C, Yang J, Chaudhry MT, Wang S, et al. Quercetin, inflammation and immunity. Nutrients 2016;8:167-81.

37. Carullo G, Cappello AR, Frattaruolo L, Badolato M, Armentano B, Aiello F. Quercetin and derivatives: useful tools in inflammation and pain management. Future Med Chem 2017;1:79-93.

38. Lee HN, Shin SA, Choo GS, Kim HJ, Park YS, Kim BS, et al. Antiinflammatory effect of quercetin and galangin in LPS-stimulated RAW264.7 macrophages and DNCB-induced atopic dermatitis animal models. Int J Mol Med 2018;41:888-98.

39. Park EJ, Kim JY, Jeong MS, Park KY, Park KH, Lee MW, et al. Effect of topical application of quercetin-3-0-(2"-gallate)- $\alpha$-lrhamnopyranoside on atopic dermatitis in $\mathrm{NC} / \mathrm{Nga}$ mice. J Dermatol Sci 2015;77:166-72. 